\title{
25 Research Square \\ Switching off key enzyme could make cancer cells more visible to immune system
}

\author{
Yu Wang \\ Qingguo Sun \\ Ning Mu \\ Xiaoyang Sun \\ Yingying Wang \\ Songqing Fan \\ Ling Su \\ Xiangguo Liu
}

\section{Video Byte}

Keywords: Cell Communication and Signaling, PD-L1, USP22, CSN5, deubiquitination, immune checkpoint blockade therapy, ICBT, immunotherapy, non-small cell lung cancer, NSCLC, chemotherapy, PD-1, T cell, deubiquitinating enzymes, DUBs, ubiquitin-specific peptidase 22, COP9 signalosome 5, immunosuppression, siRNA, antigen

Posted Date: November 3rd, 2020

DOI: https://doi.org/10.21203/rs.3.rs-102136/v1

License: (c) (i) This work is licensed under a Creative Commons Attribution 4.0 International License. Read Full License 


\section{Abstract}

PD-L1 is a powerful cloaking protein for cancer cells. Abundant PD-L1 on their surface binds with the receptor PD-1 on immune cells. The PD-L1/PD-1 axis transfers inhibitory signaling to the immune system that the cancer cells pose no danger. How this cloaking process unfolds has remained unclear. Now, researchers report the prominence of the enzyme USP22. USP22 is overexpressed in malignant tumors of several types, including those of the lung. Initial experiments on human lung cancer cells showed that USP22 might regulate PD-L1. A closer look revealed that USP22 deubiquitinated and stabilized PD-L1. USP22 enlists the help another protein, CSN5, to stabilize PD-L1. By inhibiting USP22 genetically, researchers could suppress the formation of tumors. Targeting USP22 in the clinic could therefore be one way to decloak cancer cells and make them vulnerable to existing anti-cancer therapies. 